\title{
FABRICATION AND MEASUREMENT OF LOW WORKFUNCTION CESIATED DISPENSER PHOTOCATHODES*
}

\author{
N. Moody, D. W. Feldman, P. G. O'Shea, Univ. of Maryland, College Park, MD 20742 \\ K. L. Jensen ${ }^{\#}$, Naval Research Laboratory, Washington, DC 20375
}

\section{Abstract}

A requirement for high power free electron lasers (FEL) is a high current, high quality electron beam. This is achieved using a laser-switched photocathode. Even state-of-the-art high, efficiency photocathodes suffer from short lifetime, because contamination and evaporation processes damage the surface monolayer of photosensitive material. This research seeks to improve both efficiency and lifetime through a novel dispenser cathode, where the photosensitive layer is regenerated in situ. During regeneration, photosensitive material from a sub-surface reservoir diffuses to the surface through microscopic pores to repair the damaged layer. The goal of this research is to design and prototype a robust, lowworkfunction regenerable photocathode which exhibits long lifetime and high efficiency.

\section{INTRODUCTION}

Photoemission is the preferred emission method for applications such as free electron lasers where beam quality is critical [1]. Such beams must have temporal and spatial characteristics that are most easily imparted at the cathode itself. This is achieved by using a photocathode, where short pulses from a drive laser are synchronized with accelerating fields at the cathode to produce precise bunches of charge, as shown in figure 1. The time structure of the electron beam matches that of the drive laser, allowing pulse shape to be imparted directly at the cathode. The beam is then accelerated to relativistic energies with its initial shape and quality intact.

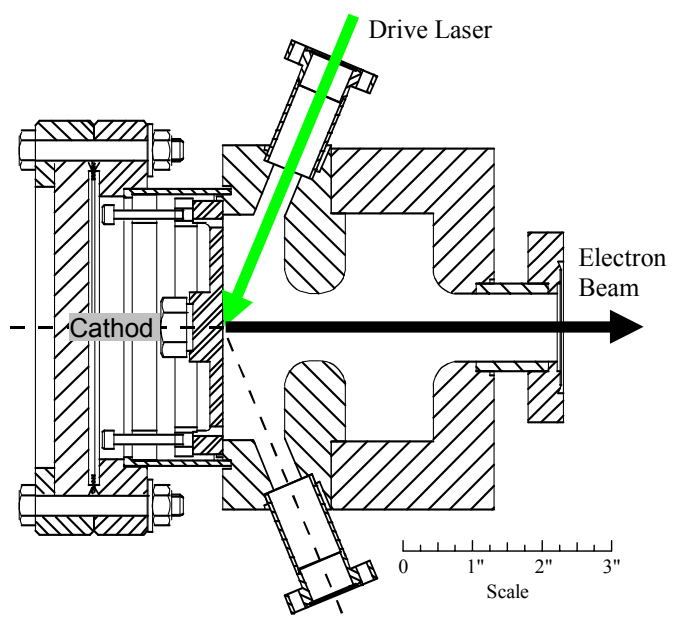

Figure 1: Photoinjector schematic.

\footnotetext{
*Work supported by Joint Technology Office (JTO), Office of Naval Research (ONR)
}

An important characteristic of photocathodes is quantum efficiency (QE) - the ratio of emitted electrons to incident photons. A goal of dispenser cathode research is to dramatically lengthen the lifetime of a photocathode, while maintaining sufficiently high quantum efficiency. Lifetime is a crucial characteristic for cathodes in FELs because it determines the duty cycle and down-time of the accelerator.

\section{Cesium on Tungsten}

This project's first undertaking was to understand and predict the photoemission process of cesiated tungsten as a starting point for designing more complex cathode systems. Cesium and other alkali metals are known to reduce the workfunction of the substrate onto which they are evaporated [2],[3]. Qualitatively, this is due to the fact that the cesium atoms give up their single valence electron and reside on the surface as a positive charge, as shown in figure 2 .

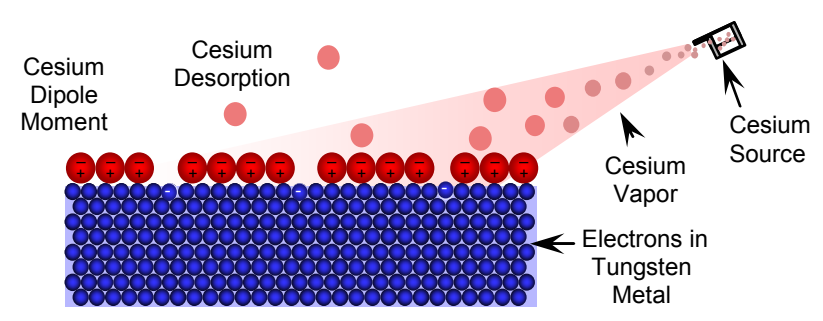

Figure 2: Cesium evaporation onto tungsten.

The effective dipole moment set up by these charges at the surface assists the electron in crossing the barrier potential. Workfunction is the amount of energy that must be imparted to an electron such that it can escape from the metal to vacuum. The reduction in workfunction is sufficient to allow some cesiated compunds to perform reasonably well as photoemitters at green and blue wavelengths [4].

\section{Predictive Theory}

A theory to account for the process of photoemission from cesiated metals has been developed at the Naval Research Laboratory [5]. In a collaborative effort with NRL, this theory has been compared with experimental results in this project and found to agree exceedingly well. Using this theory, the photoemission properties of dispenser cathodes can be predicted for various surface substances and geometries. Therefore, the experimental results gleaned thus from this program, together with the NRL theory, provide a sound starting point for the 
development of alkali-metal based dispenser photocathodes.

\section{EXPERIMENT}

The UHV fabrication system consists of a four-way cross housing the cesium evaporation sources and the anode / cathode assembly. Quantum efficiency is monitored by shining a stable diode laser (figure 3 ) onto the cathode in the presence of an electric field while measuring the resulting photocurrent as a function of evaporated thickness or coverage. Because photocurrent in this experiment could be as small as tens of nanoamperes, significant effort was required to obtain a low-noise photocurrent signal that could yield reliable QE measurement. This signal, along with pressure, temperature, evaporated thickness, background gas composition, and anode-cathode voltage are measured and recorded using Labview integrated instrumentation.

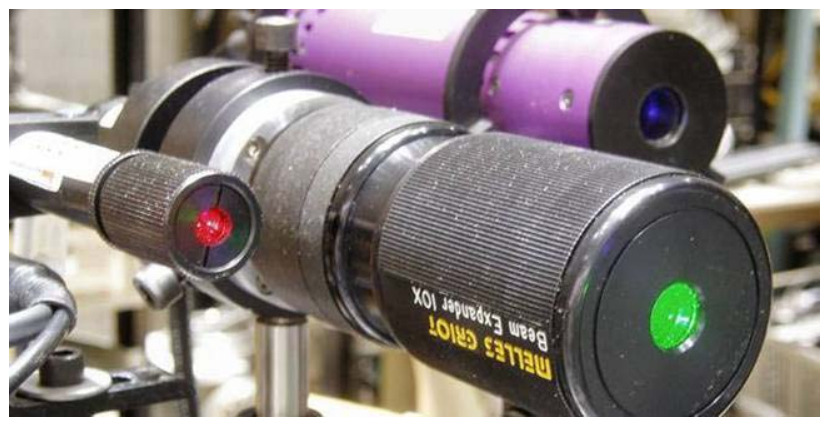

Figure 3: Solid state lasers.

Figure 4 shows the four-way cross and the evaporation system used to fabricate $\mathrm{Cs}-\mathrm{W}$ cathodes. For dispenser cathode testing, the cathode assembly will be slightly modified and the evaporation sources will be expanded to include other elements beyond just cesium. Sources of potassium and antimony will allow the fabrication of more complex and higher efficiency surface compounds on dispenser photocathodes.

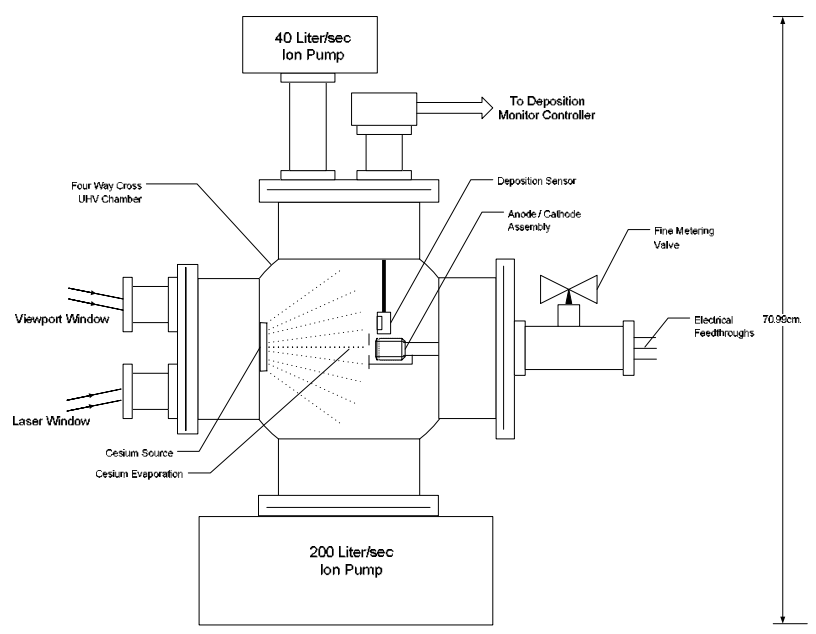

Figure 4: UVH Fabrication System.

\section{RESULTS}

\section{Bench-marking Theory}

Before embarking on the dispenser cathode work, we first studied the behavior of a cesiated tungsten sheet $0.3 \mathrm{~mm}$ thick for comparison to theoretical prediction. It was apparent that a sub-monolayer thickness of cesium on tungsten was required to reach a peak in quantum efficiency. The results were obtained in two ways: 1.) starting with bare tungsten and measuring QE as cesium was slowly added, and 2.) starting with more than a monolayer thickness of cesium and slowly removing cesium via evaporation until reaching bare tungsten.

Both methods showed in repeated experiments that QE peaks at $60-70 \%$ coverage, in very close agreement with the theory developed by K. Jensen [5]. Experimental data and theoretical predictions are plotted together in figure 5.

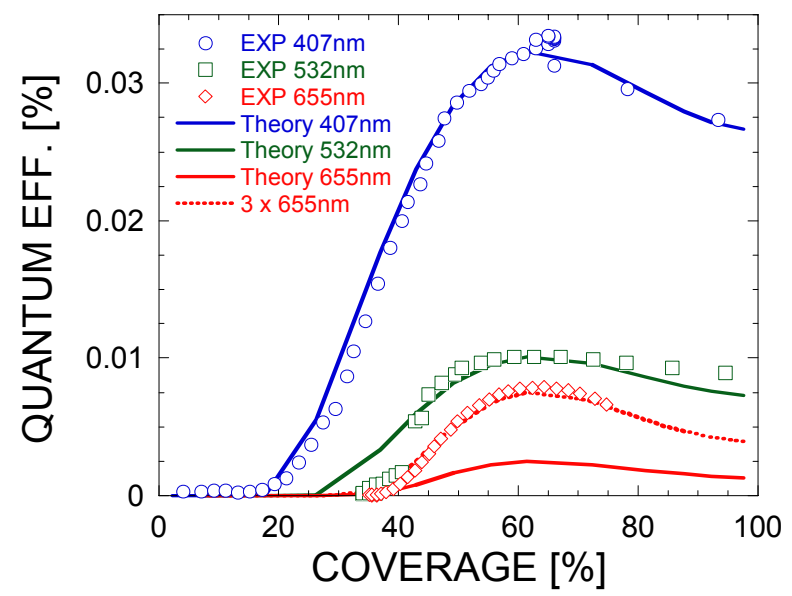

Figure 5: QE vs. coverage at various wavelengths.

This theory uses a hard-sphere model of surface atoms to calculate the changes in workfunction due to a dipole moment at the surface. It accounts for the QE versus coverage behavior in nearly every way using only first principles and standard physical assumptions [6]. The crucial result of both theory and experimentation is the relationship between surface coverage (of cesium in this case) and quantum efficiency. Notice that for blue and green, in figure 5, the theory and experiment agree exceedingly well. Matters become more complicated for red light, because the photon energy is approaching the workfunction. Near the barrier height, the photoemission process is very sensitive to any changes on the surface and it is quite possible that not everything that matters at such low energies has been properly accounted for. Nevertheless, multiplication (of the theoretical prediction for red light) by a factor of 3 seems to bring it back into agreement with experiment. Further investigation is required to determine the need for this scaling factor. Aside from this simple change, no other adjustments were made to the theoretical predictions to obtain the agreement shown. 
The peak QE which occurs at all wavelengths corresponds to a fraction of a monolayer coverage and is thus a relative indicator of the amount of cesium remaining on the surface. This measurement will serve as a crucial diagnostic in the dispenser cathode phase because it allows estimation of remaining cesium given changes in quantum efficiency.

\section{Dispenser Cathode Fabrication}

The process of atomic diffusion through irregular, microscopic pores and across an uneven surface is complicated. Research thus far suggests, however, that rejuvenation of the cathode surface is indeed possible and measurement techniques (including the QE vs. coverage relationship) have been developed to measure this effect.

Figure 6 shows a simplified schematic of a dispenser photocathode: a stainless steel canister is filled with cesium chromate powder and laser welded to a sintered or laser-drilled tungsten disk on the top. A prototype dispenser photocathode has recently been fabricated and is shown to scale in figure 7. During gentle heating, the cesium chromate is reduced to elemental cesium, which should diffuse to the surface in a controlled manner through the microscopic pores in the tungsten disk.

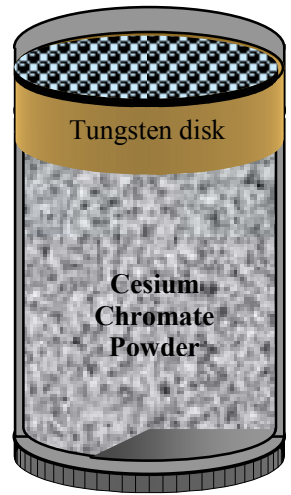

Figure 6 : Dispenser cathode schematic.

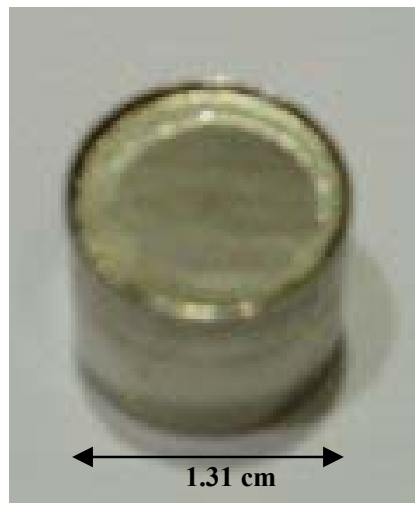

Figure 7: Prototype dispenser cathode.

\section{Bench-marking Theory}

Over the life of the cathode, cesium will leave the surface due to evaporation or ion bombardment, but periodic heating should cause cesium to again diffuse to the surface and repair the damage. The diffusion rate will be adjusted by varying porosity of the tungsten disk, until a desired balance is achieved between cesium leaving from and arriving at the surface.

\section{CONCLUSION}

\section{Future Work}

Work in the immediate future involves:

- Comparing the performance of sintered vs. laserdrilled tungsten disks,

- Developing a safe outgassing procedure for first time dispenser cathode use,

- Predict optimum porosity and compare with experiment,

- Precision temperature control for rejuvenation procedure.

After an activation procedure has been established and proven in our simple chamber, the prototype dispenser cathode will be tested inside a working RF gun to evaluate its actual performance and lifetime.

\section{Summary}

Free electron lasers show promise of becoming a nextgeneration light source because they can theoretically generate tunable radiation at currently unattainable wavelengths and power levels. A key technology for the development of FELs is the photoinjector, where the electron beam is generated. This research is motivated by the need for robust, long lifetime photocathodes with high quantum efficiency at visible wavelengths. The solution pursued in this research is a dispenser cathode, whose photoemission properties may be restored even after damage and contamination, allowing significant improvement in overall cathode lifetime.

\section{REFERENCES}

[1] S. H. Kong, J. Kinross-Wright, D. C. Nguyen, R. L. Sheffield, "Photocathodes for free electron lasers," Nucl. Instr. And Meth. in Phys. Res., A 358 (1995) 272-275.

[2] C.-S. Wang, "High photoemission efficiency of submonolayer cesium-covered surfaces," Jour. Appl. Phys. vol. 48, no. 4, April, 1977, pp. 1477-1479.

[3] E. P. Gyftopoulos, J. D. Levine, "Work function variation of metals coated by metallic films," Jour. Appl. Phys. vol. 33, no. 1, January, 1962, pp. 67-73.

[4] N. A. Moody, "Fabrication and Measurement of Cesiated Tungsten Photocathodes," Masters Thesis, ECE Dept, Univ. of MD, 2003.

[5] K. Jensen, D. Feldman, D. Demske, P.O'Shea, "Thermal and Field Enhanced Photoemission: Comparison of Theory to Experiment," Proc. FEL Conf, 2004, pp. 574-577.

[6] K. L. Jensen, D. W. Feldman, N. Moody, P. G. O'Shea, "Photoemission from Low Workfunction Coated Metal Surfaces: A Comparison of Theory to Experiment," PAC, Knoxville, TN (May, 2005). 